\title{
¿Cómo es la salud mental de los hijos de madres deprimidas consultantes a servicios de atención primaria?
}

\author{
Rosemarie Fritsch $M^{1}$, María Elena Montt $S^{1}$, Jaime Solís $\mathbf{~}^{1}$, \\ Daniel Pilowsky², María Graciela Rojas $C^{1}$. \\ Psychopathology and social \\ functioning among offspring \\ of depressed women
}

Background: Offspring of depressive parents have two times more risk of developing a depression, other psychiatric diseases or a poor social functioning. Aim: To assess psychopathology and social functioning among offspring of currently depressed mothers. Material and methods: We enrolled 290 depressed mother-child pairs in five primary-care clinics in Santiago. A two-stage screening process to identify female primary-care patients with current major depressive illness with children aged 6-16 years, was used. All eligible and consenting patients were asked to complete the general health questionnaire (GHQ-12). Those scoring 5 or more were invited to participate in a baseline assessment. The final sample consisted of 290 mother-child pairs. Patients with a current DSM-IV diagnosis of major depression were eligible unless they had current psychotic symptoms, imminent suicide risk, history of mania, or current alcohol abuse. Child psychopathology was assessed with the Child Behavior Checklist (CBCL), a highly reliable and widely used parent-rated checklist to assess competencies and behavioural and emotional problems in children 4 to 18 years of age. Results: Fifty percent (95\% confidence interval (CI): 43.9-55.7) of children had overall CBCL psychopathology scores in clinical range. Internalizing symptoms were more prevalent than externalizing symptoms (62.2\% [95\%CI: 56.3-67.8] and 35.7\% [95\%CI: 30.2-41.5]. Conclusions: A large proportion of children of depressed poor mothers attending primary care clinics in Chile, had psychopathological symptom scores in the clinical range, with a predominance of internalizing symptoms. These results are similar to those previously reported in the United States of America (Rev Méd Chile 2007; 135: 602-12).

(Key words: Depression; Infant behavior; Psychopathology)

Recibido el 8 de marzo, 2006. Aceptado el 30 de octubre, 2006.

Trabajo financiado por FONDECYT \# 1040432.

${ }^{1}$ Hospital Clínico, Facultad de Medicina, Universidad de Chile. Santiago de Chile. ${ }^{2}$ Columbia University and the New York State Psychiatric Institute, New York, USA.

Correspondencia a: Rosemarie Fritsch M. Avda. La Paz 1003,

Recoleta. Fono: 9788614. E mail: rfritsch@redclinicauchile.cl 
$\mathrm{L}$ os problemas de salud mental, en particular los trastornos depresivos, son un gran problema de salud pública tanto en los países desarrollados como en vías de desarrollo por su alta prevalencia y el grado de discapacidad que producen. Los trastornos psiquiátricos afectan especialmente a las mujeres y en particular a las de estratos socioeconómicos más bajos ${ }^{1-4}$. Uno de los hallazgos más consistentes de la epidemiología psiquiátrica es que las mujeres tienen un riesgo casi dos veces más alto que los hombres de sufrir trastornos depresivos. Las razones para explicar este fenómeno no son del todo claras. En los Estados Unidos de Norteamérica se ha calculado que 5\% a $14 \%$ de las mujeres tendrán un episodio depresivo en algún momento de su vida, en comparación con $2 \%$ a $4 \%$ de los hombres ${ }^{5}$. El estudio epidemiológico llevado a cabo en la población adulta del Gran Santiago por Araya y colaboradores, informó 8\% de prevalencia de episodio depresivo en las mujeres y $2,7 \%$ en los hombres ${ }^{4}$. Al comparar los resultados de esta investigación con los de las ciudades del Great Britain National Survey of Psychiatry Morbidity, se encuentra que la diferencia entre los sexos es significativamente más marcada en Chile, mostrando un mayor riesgo relativo de la mujer chilena en comparación con la británica 6 .

Las diferencias en la prevalencia de depresión entre hombres y mujeres se encuentran en distintas culturas $^{7}$ y se ha demostrado que ésta comienza alrededor de los 13 a 15 años y se mantiene a través de la vida. Existe un aumento significativo de la incidencia de depresión en la mujer durante los años en edad fértil, disminuyendo después de los 45 años 7 . Por lo tanto, los efectos negativos de la depresión de la mujer en el funcionamiento familiar impactan en la etapa más sensible de la crianza de los hijos ${ }^{8}$.

Un número importante de estudios realizados en Estados Unidos de Norteamérica y Europa, han demostrado que los hijos de padres depresivos tienen el doble de riesgo de desarrollar tanto una depresión como otros trastornos psiquiátricos y menor funcionamiento social ${ }^{9-12}$. Los trastornos que se observan en los hijos varían de acuerdo a la edad: en los niños en edad escolar predominan los trastornos de conducta y ansiosos, en los adolescentes se observa depresiones, en los adultos jóvenes abuso de substancias y depresiones en la adultez ${ }^{13}$.
En Santiago, se ha estudiado la salud mental de población escolar básica (6 y 11 años), encontrando una prevalencia de $15 \%$ de problemas emocionales y conductuales ${ }^{14}$. En familias pobres de Santiago se encontró una asociación entre características psicológicas maternas y riesgo de salud de sus hijos, proponiendo incluir en los programas de salud infantiles la salud mental de las madres ${ }^{15}$. Sin embargo, en nuestro país no se ha estudiado específicamente la salud mental de hijos de mujeres deprimidas.

El objetivo de esta publicación es describir la salud mental de hijos de madres deprimidas y de nivel socioeconómico medio bajo y bajo, que consultan a servicios de salud de atención primaria. Los resultados aquí expuestos son parte de un ensayo clínico aleatorio que evalúa la efectividad de un tratamiento farmacológico monitorizado a mujeres deprimidas que consultan a la atención primaria y que viven con hijos en edad escolar.

\section{Metodología}

El estudio se llevó a cabo en 5 consultorios de atención primaria de la Región Metropolitana. Se seleccionaron mujeres consultantes que cumplieran con los criterios de inclusión-depresión mayor, edad entre 18 y 70 años, con al menos 1 hijo entre 6 y 16 años viviendo con ella- y que no presentaran criterios de exclusión -tratamiento farmacológico para la depresión en los últimos 3 meses, abuso de alcohol o drogas, antecedente de enfermedad bipolar, sintomatología psicótica actual o en el pasado, embarazo o condición discapacitante mental o física que le impidiera participar en el estudio-.

En la sala de espera, se realizó un tamizaje usando el Cuestionario de Salud de Goldberg (GHQ-12). Este es un instrumento breve autoaplicado para medir malestar psíquico y que ha sido validado y usado en nuestro país en investigaciones anteriores llevadas a cabo en la atención primaria, describiéndose una sensibilidad de $78 \%$ y un valor predictivo de $55 \% 16$.

Quienes hubiesen obtenido en un primer contacto un puntaje mayor a 4 , fueron citadas para una segunda evaluación en dos semanas. Aquellas que persistieron con un puntaje mayor a 4 puntos fueron 
invitadas a participar en un diagnóstico basal, previa firma de un consentimiento informado.

En el diagnóstico basal de las mujeres deprimidas, se utilizó un cuestionario que inclúa la Mini International Neuropsychiatric Interview (MINI), una entrevista diagnóstica estructurada, de corta duración, que explora de manera estandarizada cada uno de los criterios necesarios para el establecimiento de los diagnósticos principales de las Clasificaciones Internacionales de las Enfermedades Mentales (Organización Mundial de la Salud, 1993, DSM-IV). Puede ser utilizado por clínicos, especialistas o no, que hayan recibido una formación previa ${ }^{17}$. Estudios de validez y de confiabilidad se han realizado comparando la MINI con el SCID-P para el DSM-III-R y el CIDI (una entrevista estructurada desarrollada por la Organización Mundial de la Salud para entrevistadores no clínicos para la CIE-10). Los resultados de estos estudios demuestran que la MINI tiene una puntuación de validez y confiabilidad aceptablemente alta, pero puede ser administrada en un período de tiempo mucho más breve (promedio de $18,7 \pm 11,6 \mathrm{~min}$, media $15 \mathrm{~min}$ ) que los instrumentos mencionados.

Una vez que la madre fue incluida en el estudio, se escogió un hijo al azar utilizando el método de Kish, de tal forma que cada hijo tenía la misma probabilidad de ser escogido en forma independiente del número de hijos.

Con el objeto de valorar la depresión materna se administró la Escala de Depresión de Hamilton (HRSD). Estudios en la atención primaria en Chile indican que este instrumento presenta una buena confiabilidad y validez convergente, en comparación con otras escalas de depresión ${ }^{18}$. Se midió la intensidad de la sintomatología depresiva mediante la escala de depresión de Hamilton, de 17 ítems, en su versión castellana validada ${ }^{19}$. Los puntajes obtenidos en esta escala pueden ir desde 0 hasta 52. Para este instrumento se han establecido los siguientes rangos de severidad: depresión severa (25 o más puntos), depresión moderada (sobre 17 puntos), depresión leve (sobre 6 puntos) y ausencia de depresión (6 puntos o menos $)^{20}$.

La sicopatología de los menores se investigó a través del informe de la madre del niño o adolescente y de un evaluador externo, contando con criterios diferentes que posteriormente fueron correlacionados. Para esto se aplicaron los siguientes instrumentos.

Child Behavior Check-list (CBCL) (Listado de comprobación de la conducta del niño), contestado por la madre. Este instrumento, en su versión para padres, consiste en un formulario estandarizado para registrar los problemas comportamentales y competencias sociales de niños entre 4 y 16 años. Incluye 20 ítems referidos a competencias sociales (desempeño escolar, relaciones sociales y participación en actividades) y 118 ítems de problemas comportamentales categorizados del siguiente modo: $0=$ no es cierto, $1=$ es cierto algunas veces o de cierta manera, 2 =muy cierto o a menudo cierto. El puntaje total se obtiene a partir de la suma de los parciales. Cuanto más alto es el puntaje, mayor es el nivel de trastorno del niño. Se pueden obtener escalas amplias o estrechas -síndromes-. El inventario requiere, según su autor, un nivel de lectura correspondiente a 5o básico, es autoadministrado y toma entre 15 y 20 min. La buena confiabilidad y validez establecida por sus autores, han sido confirmadas transculturalmente, incluyendo países de habla hispana como Chile, Puerto Rico y España $^{21,22}$. El CBCL es un instrumento fiable para distinguir niños con sicopatología de población general. Posee una sensibilidad de $67 \%$ y una especificidad de $74 \%$. Genera un puntaje general estandarizado según edad y sexo de sicopatología compuesto por 2 subdominios: síntomas internalizantes (depresión, ansiedad) y externalizantes (trastornos disruptivos) (rango 0 a 100). Puntajes mayores reflejan mayor presencia y severidad de síntomas. Puntajes mayores de 70 son considerados en rango clínico 23 .

Escala Breve de Apreciación Psiquiátrica para Niños y Adolescentes (EBA-NA) realizada por psicólogos especialmente entrenados. Se utiliza para investigaciones epidemiológicas de tratamiento, realizar diagnóstico y para seguimientos longitudinales. Es una escala multidimensional, que describe la presencia y severidad de 21 síntomas y genera 7 factores y un puntaje total para la presencia de patología psiquiátrica. Se utiliza desde los 3 a los 18 años de edad. Posee un buen nivel de confiabilidad y validez ${ }^{24,25}$. Para la medición de sintomatología psiquiátrica se utiliza un punto de corte de $8 / 9$. 
Escala Autoinforme de Ansiedad (AA), es un tamizaje de síntomas ansiosos. Tiene una buena consistencia interna, validez y confiabilidad. Consiste en un autorreporte de síntomas que se dividen en 5 grupos que incluyen la fobia escolar, fobia social, ansiedad de separación, ansiedad, generalizada, trastorno pánicosomatización. Consta de 41 ítems que se puntúan de 0 a 2, con un punto de corte de 35 puntos $^{26,27}$.

Cuestionario Depresión Infantil (CDI) de Kovaks. Cuestionario de autorreporte de síntomas de depresión, que consta de 27 ítems, puntuados de 0 a 2 , con un punto de corte de 181. Se evalúan síntomas cognitivos, afectivos y conductuales de depresión en menores de 8 a 16 años. Posee una alta consistencia interna y fue validado en Chile ${ }^{28,29}$.

Con el objeto de evaluar la relación de pareja se administró a las mujeres deprimidas, la escala DAS (Dyadic Adjustment Scale) ${ }^{30}$ abreviada, que mide la calidad de la adaptación en la vida de pareja. Este es uno de los instrumentos más usados en investigación familiar y de pareja ${ }^{31}$. Se seleccionaron los ítems que tenían la mayor consistencia interna. Tiene un puntaje de 0 a 33, correspondiendo el mayor a un peor ajuste en la pareja $^{32}$.

Con el objeto de explorar la funcionalidad familiar, se le administró a la madre el APGAR familiar. Este instrumento mide adaptabilidad, cooperación, desarrollo, afectividad y capacidad resolutiva ${ }^{33}$. Su puntaje fluctúa entre 0 y 10 , correspondiendo el mayor a una mejor funcionalidad familiar. De acuerdo con los puntajes es posible clasificar el funcionamiento familiar en funcional y disfuncional, y este último en leve y grave.

Las bases de datos fueron confeccionadas en el paquete estadístico SPSS $11.0^{34}$, para luego realizar los análisis en el programa STATA 8.0 35 . Para las variables continuas, se calcularon los promedios y respectivos intervalos de confianza. Para las variables categóricas, se calcularon las proporciones y sus respectivos intervalos de confianza (IC: 95\%). Con el objeto de determinar la participación de múltiples factores en el riesgo de aparición de sicopatología infantil se calcularon las razones de disparidad ajustadas a las variables analizadas. Todos los niveles de significación fueron fijados en $95 \%$ de confiabilidad.

\section{Resultados}

Descripción de la muestra. La muestra estuvo constituida por 290 parejas madre-hijo.

La edad promedio de las madres fue de 37,4 años $(36,6-38,1)$. La mayoría de ellas había cursado la enseñanza media (68,3\%). El 20,6\% sólo había cursado la enseñanza básica, y 11,0\% contaban con estudios superiores. El $76,1 \%$ de las mujeres vivía con una pareja $(54,6 \%$ era casada y $21,5 \%$ conviviente). El 23,9\% vivía sin pareja (13,4\% eran separadas, 9,3\% solteras y 1,2\% viudas). El número promedio de hijos era $2,7(2,5-2,8)$.

Al momento de ser incluidas en el estudio, las mujeres tenían un puntaje de 19,1 puntos (18,619,7) en la escala HDRS. El 36,9\% (30,7-43,8) tenía una depresión leve, 49,6\% (42,4-57,6) moderada y 13,6\% (10,0-18,1) severa. El 56,2\% de ellas tenía antecedentes de episodios depresivos previos.

Los hijos tenían una edad promedio de 10,5 años $(10,2-10,9)$. El 50,3\% (44,4-56,2) eran mujeres y $49,6 \%(43,8-55,6)$ hombres (Tabla 1$)$.

No se encontró diferencias estadísticamente significativas entre los puntajes obtenidos por las madres en el HDRS y el nivel educacional y estado civil $(\mathrm{F}=0,697 ; \mathrm{p}=0,595)(\mathrm{F}=0,073 ; 0,990)$.

Salud mental de los hijos. El 49,8\% de los niños $(43,9-55,7)$ presentaba puntajes totales en el CBCL en rango clínico. El 62,2\% tenía síntomas internalizantes y $35,7 \%$ externalizantes. Los subdominios internalizantes (ansioso-depresivo, inhibido, quejas somáticas) se distribuían en forma relativamente homogénea entre $45,4 \%$ y $48,8 \%$. Dentro de los externalizantes, predominaba la agresividad $(40,9 \%)$ frente a la conducta antisocial (21,3\%) (Tabla 2).

La proporción de niños con puntaje en el AA en rango clínico fue de $62,7 \%(56,8-68,3)$, en el CDI fue de $25,9 \%(20,9-31,6)$ y el EBA de $51,9 \%(46,0-57,8)$.

Armonía matrimonial y funcionalidad familiar. El puntaje promedio obtenido en la escala de armonía matrimonial fue de 16,6 y en el APGAR familiar de 5,8. El 56,6\% presentaba disfunción familiar según el mismo instrumento.

Salud mental de los hijos y posibles asociaciones. De acuerdo con los resultados obtenidos en los análisis de regresión logística, la única variable que se asoció significativamente y en forma independiente con la 
Tabla 1. D escripción de la muestra ( $\mathrm{N}=290)$

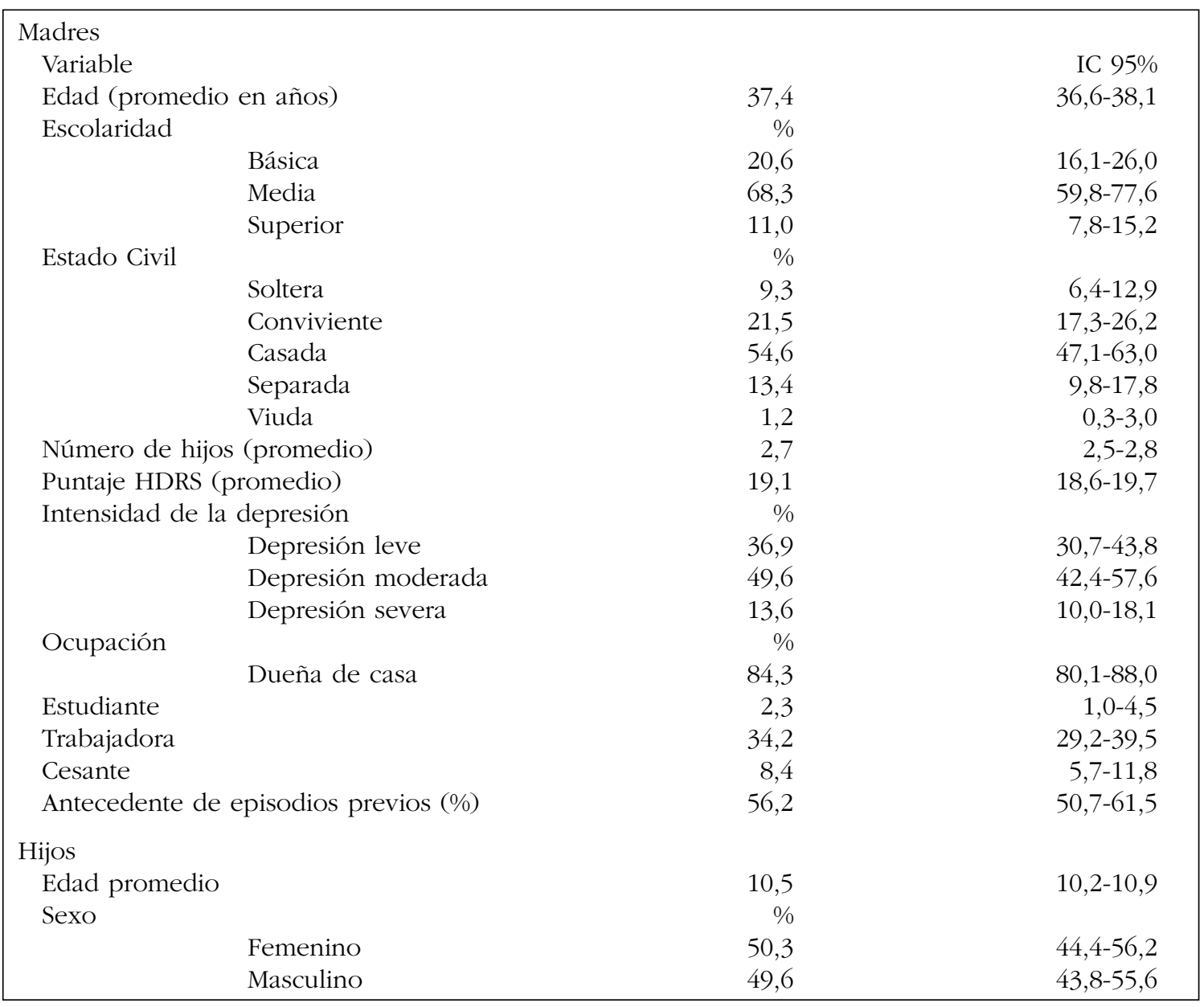

Tabla 2. Proporción de niños con sintomatología en rango clínico, según CBCL (N =290)

\begin{tabular}{|lcc|}
\hline & $\%$ & IC $95 \%$ \\
\hline Sintomatología psiquiátrica global & 49,8 & $43,9-55,7$ \\
Síntomas internalizantes & 62,2 & $56,3-67,8$ \\
Síntomas externalizantes & 35,7 & $30,2-41,5$ \\
\hline
\end{tabular}

salud mental global del niño en rango clínico fue el grado de depresión de la madre medido a través de la escala de Hamilton (Tabla 3).

Hubo una correlación estadísticamente significativa entre el puntaje total obtenido en el CBCL y los puntajes de las escalas autoadministradas: Cuestionario de Depresión Infantil (Correlación de Pearson: 0,179; $\mathrm{p}=0,003)$ y Autorreporte de Ansiedad (Correlación de Pearson: 0,198; p $=0,001)$, y las obtenidas a través de las observacio- 
nes del evaluador: Escala Breve de Apreciación Psiquiátrica para Niños y Adolescentes (Correlación de Pearson: 0,214; $\mathrm{p}=0,000$ ) (Tabla 4).
No hubo correlación entre el puntaje obtenido por la madre en el HDRS y los puntajes de las escalas autoadministradas Cuestionario de Depre-

Tabla 3. Sintomatología psiquiátrica de hijos y posibles factores asociados*

\begin{tabular}{|c|c|c|c|c|c|c|c|}
\hline \multirow{2}{*}{\multicolumn{2}{|c|}{ Variable }} & \multicolumn{2}{|c|}{$\begin{array}{c}\text { Sintomatología } \\
\text { psiquiátrica global }\end{array}$} & \multicolumn{2}{|c|}{$\begin{array}{c}\text { Síntomas } \\
\text { internalizantes }\end{array}$} & \multicolumn{2}{|c|}{$\begin{array}{c}\text { Síntomas } \\
\text { externalizantes }\end{array}$} \\
\hline & & R.D.(IC 95\%) & $\mathrm{p}$ & R.D.(IC 95\%) & $\mathrm{p}$ & R.D.(IC 95\%) & $\mathrm{p}$ \\
\hline \multicolumn{8}{|l|}{ Infantiles } \\
\hline Edad & & $1,0(0,9-1,1)$ & 0,847 & $1,0(0,9-1,1)$ & 0,424 & $1,0(0,9-1,1)$ & 0,838 \\
\hline \multirow[t]{2}{*}{ Sexo } & hombre & 1 & & 1 & & 1 & \\
\hline & mujer & $0,8(0,5-1,5)$ & 0,584 & $1,0(0,5-1,9)$ & 0,961 & $0,7(0,4-1,3)$ & 0,309 \\
\hline \multicolumn{8}{|l|}{ Maternas } \\
\hline Edad & & $1,0(1,0-1,1)$ & 0,192 & $1,0(0,9-1,0)$ & 0,733 & $1,0(1,0-1,1)$ & 0,778 \\
\hline \multirow[t]{3}{*}{ Educación } & superior & 1 & & 1 & & & \\
\hline & secundaria & $0,7(0,2-2,2)$ & 0,581 & $1,0(0,3-3,3)$ & 0,938 & $0,7(0,2-2,1)$ & 0,494 \\
\hline & primaria & $1,0(0,4-2,6)$ & 0,968 & $1,5(0,6-4,0)$ & 0,378 & $0,6(0,2-1,7)$ & 0,367 \\
\hline \multirow[t]{4}{*}{ Estado civil } & soltera & 1 & & 1 & & 1 & \\
\hline & conviviente & $0,9(0,2-4,0)$ & 0,886 & $2,0(0,4-9,5)$ & 0,398 & $0,7(0,1-3,0)$ & 0,592 \\
\hline & casada & $0,8(0,2-3,2)$ & 0,711 & $1,8(0,4-8,3)$ & 0,442 & $0,5(0,1-2,2)$ & 0,350 \\
\hline & separada & $0,6(0,1-4,9)$ & 0,635 & $0,7(0,1-6,4)$ & 0,784 & $0,4(0,0-3,7)$ & 0,415 \\
\hline \multirow[t]{3}{*}{ Ocupación } & dueña de casa & $1,4(0,3-5,9)$ & 0,656 & $4,1(0,9-18,4)$ & 0,066 & $0,7(0,2-3,1)$ & 0,652 \\
\hline & estudiante & $3,6(0,4-30,0)$ & 0,231 & $5,4(0,4-65,3)$ & 0,184 & $1,9(0,2-14,3)$ & 0,555 \\
\hline & trabajadora & $0,8(0,2-2,7)$ & 0,675 & $1,4(0,4-5,0)$ & 0,601 & $0,5(0,1-1,7)$ & 0,241 \\
\hline \multicolumn{2}{|c|}{ Número de ocupaciones } & $0,6(0,2-1,9)$ & 0,349 & $0,4(0,1-1,6)$ & 0,205 & $1,0(0,3-3,4)$ & 0,973 \\
\hline \multicolumn{2}{|c|}{ Número de hijos } & $1,1(0,8-1,5)$ & 0,569 & $1,5(1,0-1,9)$ & 0,063 & $1,1(0,8-1,4)$ & 0,638 \\
\hline \multicolumn{2}{|c|}{ Depresión recurrente no } & 1 & & 1 & & 1 & \\
\hline \multirow{2}{*}{\multicolumn{2}{|c|}{ APGAR familiar }} & $0,8(0,5-1,5)$ & 0,541 & $0,9(0,5-1,6)$ & 0,679 & $0,8(0,4-1,4)$ & 0,445 \\
\hline & & $1,0(0,9-1,2)$ & 0,657 & $1,0(0,9-1,1)$ & 0,881 & $0,9(0,8-1,1)$ & 0,376 \\
\hline \multicolumn{2}{|c|}{ Armonía matrimonial } & $1,0(1,0-1,1)$ & 0,298 & $1,0(1,0-1,1)$ & 0,183 & $1,0(1,0-1,1)$ & 0,538 \\
\hline \multicolumn{2}{|c|}{ HDRS } & $1,1(1,0-1,1)$ & 0,035 & $1,1(1,0-1,1)$ & 0,133 & $1,1(1,0-1,1)$ & 0,049 \\
\hline
\end{tabular}

*Razones de disparidad ajustadas de niños con puntajes en rango clínico según variables maternas e infantiles.

Tabla 4. Correlación entre las respuestas en instrumentos contestados por la madre, el niño y un psicólogo

\begin{tabular}{|c|c|c|c|c|c|c|}
\hline & \multicolumn{2}{|c|}{$\mathrm{EBA}^{4}($ psicólogo $)$} & \multirow{2}{*}{$\begin{array}{l}\qquad \mathrm{AA}^{3} \text { (niño) } \\
\text { Correlación de Pearson }\end{array}$} & \multicolumn{3}{|c|}{$\mathrm{CDI}^{2}($ niño $)$} \\
\hline & Correlación de Pearson & $\mathrm{p}$ & & $\mathrm{p}$ & Correlación de Pearson & $\mathrm{p}$ \\
\hline $\mathrm{CBCL}^{1}$ (madre) & 0,214 & 0,000 & 0,184 & 0,002 & 0,171 & 0,004 \\
\hline $\mathrm{CDI}^{2}$ (niño) & 0,205 & 0,000 & 0,348 & 0,000 & & \\
\hline $\mathrm{AA}^{3}$ (niño) & 0,164 & 0,006 & & & & \\
\hline
\end{tabular}

${ }^{1}$ CBCL: Children Behaviour Check List (Cuestionario respondido por la madre). ${ }^{2} \mathrm{CDI}$ : Cuestionario de Depresión Infantil (Cuestionario respondido por el niño). ${ }^{3}$ AA: Autorreporte de Ansiedad (Cuestionario respondido por el niño). ${ }^{4} \mathrm{EBA}$ : Escala Breve de Apreciación Psiquiátrica para Niños y Adolescentes (Cuestionario respondido por evaluador). 
sión Infantil (Correlación de Pearson: 0,056; p =0,347) y Autorreporte de Ansiedad (Correlación de Pearson: 0,099; $\mathrm{p}=0,094)$ ni con las obtenidas a través de las observaciones del evaluador: Escala Breve de Apreciación Psiquiátrica para Niños y Adolescentes (Correlación de Pearson: -0,0400; p $=0,502$ ), lo que sugiere que la correlación encontrada con el CBCL se debe a una asociación entre el grado de depresividad de las madres y la percepción que tienen acerca de la salud mental de sus hijos.

\section{Conclusiones}

Se trata de una investigación que estudia la salud mental de hijos de madres deprimidas consultantes de la atención primaria en la ciudad de Santiago, siendo la primera comunicación científica que entrega antecedentes sobre el impacto de la patología depresiva en la progenie e incluye una muestra extensa de madres deprimidas y sus hijos.

La enfermedad depresiva tiene una alta prevalencia, especialmente entre las mujeres en edad fértil y ha sido abordada por la salud pública chilena en un programa nacional e incluida en las garantías explícitas de salud. Hasta el momento, ningún programa de tratamiento de la patología depresiva en nuestro país incluye una evaluación de los hijos, quienes pudiesen constituir un grupo de alto riesgo.

Los resultados de esta investigación indican que los hijos de madres deprimidas presentan una alta prevalencia de problemas conductuales y emocionales (49,8\%). Estas cifras son mayores a los resultados obtenidos por investigaciones llevadas a cabo en nuestro país en niños de la población general (15\%), utilizando el mismo instrumento de evaluación. Otros estudios realizados en Chile en la comunidad escolar han encontrado prevalencias de $24 \%$ y $32 \%$ de trastornos psiquiátricos ${ }^{36}$.

Investigaciones internacionales realizadas en hijos de madres depresivas, llevadas a cabo en Australia, Nueva Zelanda, Estados Unidos de Norteamérica y Reino Unido, también han encontrado una alta prevalencia de trastornos psiquiátricos en éstos (con cifras entre 41\% a 77\%) $9,37-40$ mostrando que tienen 2 a 5 veces más riesgo de presentar sicopatología, que la población general. Estas cifras son similares a las encontradas en el presente estudio. No existen publicaciones sobre estudios en hijos de padres con patología depresiva en otros países en vías de desarrollo, que nos permita comparar las diferencias que se puedan encontrar en países con distintos grados de desarrollo.

Los resultados aquí obtenidos son concordantes con otro estudio realizado en Chile en hijos de madre bipolares, en los que se encontró que $46,3 \%$ de los hijos tenía algún trastorno en la salud mental ${ }^{41}$.

Dado que el CBCL es un instrumento contestado por la madre, sus respuestas pueden estar mediatizadas por su trastorno depresivo, razón por la cual se utilizó el EBA, que fue administrado por un psicólogo entrenado. Este instrumento arrojó como resultado una prevalencia de 51,9\% de sintomatología psiquiátrica en los hijos de las madres deprimidas quienes, además, autorreportaron $62,7 \%$ de síntomas ansiosos en rango clínico, y 25,9\% de síntomas depresivos en rango clínico. Estos resultados son concordantes con $62,2 \%$ de síntomas internalizantes referidos por la madre. Se encontró una correlación significativa entre los resultados obtenidos por diferentes sujetos (madre-hijo-evaluador) lo que permite aseverar que hay concordancia, entre éstos, aunque probablemente la madre podría estar subestimando levemente la presencia de sicopatología en sus hijos, ya que ellas reportan 49,8\% de sintomatología total en el CBCL, v/s 51,9\% en el EPA, referido por el evaluador externo. Estas diferencias son estadísticamente significativas $\left(\mathrm{chi}^{2}=9,1\right.$; $\mathrm{p}=0,002$ ). No se confirma la hipótesis que la depresión de la madre podría estar influyendo en tener una percepción más negativa de sus hijos, en el sentido de sobreestimar sus síntomas psiquiátricos y dificultades socio-emocionales.

Aún no están claros los mecanismos por los cuales los hijos de madres deprimidas desarrollan alteraciones en su salud mental. Se han intentado explicar a través de diferentes enfoques teóricos y diferentes hipótesis. De acuerdo con la teoría de sistemas $^{42,43}$, cuando cambia una parte del sistema, éste cambia en su totalidad. De acuerdo con estos postulados, una madre que padece una depresión facilitaría un reajuste de los demás miembros de la familia a través de interacciones recurrentes y repetitivas, siendo a la vez modificada por éstas. Al respecto, se ha mostrado que las madres deprimidas, en comparación con grupos controles, tienen más interacciones negativas, menos positivas, menos vocalizaciones, menos acercamiento y más conductas evitativas ${ }^{44}$. 
También se ha encontrado que en las familias con un miembro con trastorno del ánimo hay alteraciones en la expresividad emocional, con un alto criticismo y sobreinvolucración ${ }^{45}$. Estas transacciones contribuirían en la mantención y recaídas de la enfermedad. Se ha propuesto que la disfunción o discordia familiar en las familias de madres con depresión podría ser un mediador importante en la aparición de trastornos mentales en sus hijos.

Se ha estudiado la asociación entre depresión materna con dificultades en el funcionamiento familiar y en el ajuste marital, planteándose la idea que la discordia o disfunción familiar podría ser un mediador importante en la aparición de trastornos mentales en los hijos de mujeres deprimidas ${ }^{46}$. Este planteamiento no se corroboró en esta investigación, ya que no se encontraron diferencias significativas en el ajuste marital y en el funcionamiento familiar de las madres deprimidas, con hijos con problemas en la salud mental en comparación con los sanos. Si bien el promedio total obtenido en el funcionamiento familiar en las madres deprimidas correspondía al rango de disfuncionalidad $(5,8)$, concordante con las investigaciones, no se encontró una relación con la salud mental de los hijos. Esto podría explicarse por el hecho que se está comparando grupos extremos, en el que la mayoría de las mujeres deprimidas tenía dificultades familiares $(56,8 \%)$, lo que podría hacer perder el efecto de estas variables, y no permitiría discriminar entre hijos con y sin problemas en la salud mental y la presencia de discordia familiar en esta muestra.

La teoría del apego ${ }^{47}$ también ha intentado explicar las alteraciones en el desarrollo socioemocional que presentan los hijos de madres deprimidas. De acuerdo con esta teoría, la calidad del vínculo con la madre, su disponibilidad y sensibilidad hacia el hijo, sería una base fundamental para la calidad de la salud mental de éste. Las investigaciones han mostrado que las madres deprimidas tienden a desarrollar un apego inseguro, a partir del cual se construiría un modelo de relación con el entorno y consigo mismo, que predispondría al desarrollo de diversos trastornos psiquiátricos ${ }^{48}$. Las teorías neurobiológicas han contribuido a explicar las alteraciones del funcionamiento socioemocional de los hijos de madres depresivas. La madre representa un papel modulador en el desarrollo neurobiológico del SNC de sus hijos. El término "esperando la experiencia" ha sido usado con referencia al proceso que involucra la preparación del cerebro del niño para recibir tipos específicos de información desde el medio. Esta preparación está presente, principalmente, durante los primeros años de vida, en los periodos del crecimiento denominados "críticos" $\mathrm{O}$ "sensibles". Estos periodos, que son cruciales para la adquisición de informaciones sociales, afectivas y cognitivas, llevan a una estabilización y mayor proliferación de determinadas sinapsis, en detrimento de otras ${ }^{49}$. Si la madre falla en proveer al niño la protección y estímulos adecuados, las posibilidades de daño a los procesos neurobiológicos y psicológicos aumentan significativamente, ocasionando repercusiones a mediano y largo plazo ${ }^{50}$.

Se ha encontrado que los hijos de madres con depresión crónica tienen una actividad cerebral atípica, con menor actividad del lóbulo frontal y parietal. Estos cambios en la actividad cerebral se explicarían por la cualidad de la relación depresiva con su hijo en asociación con factores familiares, tal como el estrés y la discordia o disfunción marital ${ }^{44}$.

En nuestro estudio no se encontró una asociación con la cronicidad o recurrencia de la depresión y el riesgo de presentar problemas de salud mental en los hijos lo que podría explicarse por las ideas planteadas por Rutter ${ }^{51}$, respecto a que los hijos de padres con patologías crónicas podrían desarrollar mecanismos adaptativos y de resiliencia para ajustarse a esta situación vital.

La severidad de los síntomas depresivos de la madre fue la única variable que se asoció con el riesgo de enfermar en sus hijos, siendo concordante con otras investigaciones, que también han mostrado que la severidad de los síntomas de la madre podría ser la más importante en el desarrollo de trastornos psiquiátricos ${ }^{52,53}$. Es posible que la asociación entre la severidad de la enfermedad de la madre y los síntomas en los hijos, se retroalimenten mutuamente, en un circulo vicioso, así las madres se deprimen más severamente porque tiene un hijo con un trastorno en la salud mental, y a la vez la depresión de la madre agrava la sintomatología del hijo. Es necesario investigar la influencia mutua entre un hijo y una madre enferma. Al respecto es interesante referir una reciente investigación sobre depresión materna, criticismo y síntomas internalizantes y externalizantes en hijos adolescentes. Se encontró que los 
síntomas externalizantes de los adolescentes mediaban la relación entre cronicidad/severidad de la depresión y criticismo materno. Se plantea la necesidad de discutir los modelos causa-efecto en la díada madre depresiva e hijo con síntomas psiquiátricos ${ }^{54}$.

En esta muestra no se encontró diferencias por sexo en el riesgo de enfermar de los hijos de madres depresivas, lo que es concordante con los resultados encontrados en estudios nacionales en población escolar y en hijos de madres bipolares $^{9,41}$. Estos resultados no confirmarían la idea que las hijas de madres deprimidas tendrían más riesgo de enfermar que los hijos por un efecto de modeling o identificación con la madre ${ }^{46}$.

Un grupo importante de hijos presenta síntomas externalizantes $(35,7 \%)$, lo que resulta contradictorio con las teorías del aprendizaje social y con las teorías genéticas de las enfermedades, por lo que se requieren más estudios para comprender cuáles son las variables mediadoras que explican las dificultades conductuales de los hijos de madres depresivas. Las investigaciones sobre el temperamento de los hijos y la calidad de la red de apoyo, podrían contribuir a esclarecer estos factores.

En futuras investigaciones será necesario evaluar el riesgo relativo de enfermar en los hijos de madres depresivas en diferentes etapas evolutivas y precisar si hay mayor riesgo de trastornos mentales específicos en distintas estadios del desarrollo y sus posibles diferencias de género.

\section{REFERENCIA}

1. Murray C, López A. Alternative projections of mortality and disability by cause 1900-2020: Global Burden of Disease Study. Lancet 1997; 349: 1498-504.

2. Patel V, Araya R, Ludemir A, Todd C, Lima M. Women, poverty and common mental disorders in four restructuring societies. Soc Sci Med 1999; 49: 1461-71.

3. Jenkins R, Lewis G, Bebbington P et al. The national psychiatric morbidity surveys of Great Britain: initial findings from the household survey. Psychol Med 1997; 27: 775-90.

4. Araya R, Rojas G, Fritsch R, Acuña J, Lewis G. Common mental disorders in Santiago, Chile: prevalence and socio-demographic correlates. $\mathrm{Br}$ J Psychiatry 2001; 178: 228-33s.

5. NIMH Breaking Ground, Breaking Through: The
También sería relevante conocer los factores protectores y de riesgo para los trastornos mentales en estos hijos, intentando precisar que hijos tendrían más riesgo de enfermar.

Los resultados de esta investigación muestran que los hijos de madres con trastorno del ánimo se encuentran en alto riesgo de desarrollar trastornos psiquiátricos, lo que es especialmente alarmante en nuestro país, dada la alta prevalencia de esta enfermedad en las mujeres chilenas en edad fértil ${ }^{4,6,7}$. Investigaciones internacionales refieren que un tercio de las mujeres tienen la posibilidad de presentar una depresión a lo largo de la vida, especialmente las más pobres y las que no tienen pareja $^{55}$, y sus hijos tienen $61 \%$ de posibilidades de desarrollar un trastorno mental durante la niñez o adolescencia, y tiene 4 veces más posibilidades de desarrollar una enfermedad afectiva en comparación con los que tienen padres sanos ${ }^{56}$.

Dado lo anterior, se sugiere que en el programa nacional para la depresión se incorpore una evaluación y tratamiento de los hijos de los que presentan síntomas clínicos y subclínicos de alteraciones en la salud mental. Es muy posible que si no se trata a los hijos con problemas en la salud mental, se mantenga la depresión de las madres, por una influencia recíproca. Estudios incipientes de tratamiento preventivo, de corta duración, en familias con un padre con depresión, han mostrado ser eficientes en la promoción de resiliencia en éstos, la que se ha mantenido en seguimientos de hasta $21 / 2$ años ${ }^{57}$.

Strategic Plan for Mood Disorders Research of the National Institute of Mental Health. NIH Publication № 03-5121 January 2002.

6. Rojas G, Fritsch R, Araya R. Salud Mental en el Gran Santiago SERNAM 2002 Stgo. Chile.

7. The changing rate of major depression. Crossnational comparisons. Cross-National Collaborative Group. JAMA 1992; 268: 3098-105.

8. Weissman MM, Gammon GD, John K, Merikangas KR, Warner V, Prusoff BA et al. Children of depressed parents. Increased psychopathology and early onset of major depression. Arch Gen Psychiatry 1987; 44: 847-53.

9. Hammen C, Burge D, Burney E et al. Longitudinal study of diagnoses in children of women with unipolar and bipolar affective disorder. Arch Gen Psychiatry 1990; 47: 1112-17. 
10. Lieb R, Isensee B, Hofler M et al. Parental major depression and the risk of depression and other mental disorders in offspring. Arch Gen Psychiatry 2002; 59: 365-74.

11. Pilowsky DJ, Wickramaratne P, Rush AJ, Hughes C, Garber J, Malloy E et al. Children of Currently Depressed Mothers: A STAR*D Ancillary Study. Journal of Clinical Psychiatry 2006; 67: 126-36.

12. Hammen C, Brennan PA, ShiH JH. Family discord and stress predictors of depression and other disorders in adolescent children of depressed and nondepressed women. Journal of the American Academy of Child \& Adolescent Psychiatry 2004; 43: 994-1002.

13. Weissman MM, Wickramaratne P, Nomura Y et al. Families at high and low risk for depression: a 3generation study. Arch Gen Psychiatry 2005; 62: 29-36.

14. Bralic S, Seguel X, Montenegro H. Prevalence of mental disorders in the schoolchild population of Santiago de Chile. Acta Psiquiatr Psicol Am Lat 1987; 33: 316-25.

15. Araya M, Espinoza J, Zegers B, Cruchet S, Brunser O, Humphreys D ET AL. Altered maternal psychological profile as health risk factor in poor urban infants. Acta Paediatr 1996; 85: 1213-6.

16. Araya R, Wynn R, Lewis G. Comparison of two self administered psychiatric questionnaires (GHQ-12 and SRQ-20) in primary care in Chile. Soc Psychiatry Psychiatr Epidemiol 1992; 27: 168-73.

17. Sheehan DV, Lecrubier Y, Sheehan KH, Amorim P, Janavs J, Weiller E et al. The Mini-International Neuropsychiatric Interview (M.I.N.I.): the development and validation of a structured diagnostic psychiatric interview for DSM-IV and ICD-10. J Clin Psychiatry 1998; 59 Suppl 20: 22-33; 34-57.

18. Araya R, Rojas G, Fritsch R, Gaete J, Rojas M, Simon $G$ ET AL. Treating depression in primary care in low-income women in Santiago, Chile: a randomized controlled trial. Lancet 2003; 361(9362): 995-1000.

19. Ramos-Brieva JA, Cordero Villafáfila A. Validación de la versión castellana de la escala de Hamilton para la depresión. Actas Luso-Esp Neurol Psiquiatr 1986; 14: 324-34.

20. Endicott J, Cohen J, NeE J et al. Hamilton depression rating scale. Extracted from regular and change versions of the schedule for affective disorders and schizophrenia. Arch Gen Psychiatry 1981; 38: 93-103.
21. Rubio-Stipec M, Bird H, Canino G, Gould M. The internal consistency and concurrent validity of a Spanish translation of the Child Behavior Checklist. J Abnorm Child Psychol 1990; 18: 393-406.

22. Montenegro H. Salud mental del escolar: Estandarización del Inventario de Problemas Conductuales y Destrezas Sociales de T. Achenbach Santiago de Chile: CEDEP-UNICEF. 1983.

23. ACHENBACH TM. Manual for the Child Behavior Checklist/4-18 and 1991 Profile. Burlington, Vt: University of Vermont Department of Psychiatry; 1991.

24. Hughes CW, Rintelmann J, Emske GJ, López M, Mac CABEn A. Revised Anchored Version of BPRS-C por Chilhood pychiatric disorder. J Child Adolesc Psychofamcol 2001; 11: 77-93.

25. Lachar D, Randle SL, Harper Ra, Scott-Gurnell KC, Lewis KR, Santos CW et aL. The brief psychiatric rating scale for children (BPRS-C): validity and reliability of an anchored version. J Am Acad Child Adolesc Psychiatry 2001; 40 (3).

26. Birmaher B, Khetarpal S, Brent D y col. The Screen for Child Anxiety Related Emotional Disorders (SCARED): Scale Construction and Psychometric Characteristics. J Am Acad Child Adolesc Psychiatry 1997; 36: 545-53.

27. Birmaher B y col. Pschometric Properties of the Screen for Child Anxiety Related Emotional Disorders (SCARED): A Replication Study. J Am Acad Child Adolesc Psychiatry 1999; 38: 1230-6.

28. Coggiola A, Gujón P. Estandarización del Inventario de Depresión en Niños de Kovacs y Beck - CDI Tesis de grado para optar al Titulo Profesional de Psicólogo Universidad Diego Portales, Santiago. 1991.

29. Kovacs M. Children's Depression Inventory Manual. New York Multi health Systems. 1992.

30. SpANIER GB. Measuring dyadic adjustment: new scales for assessing the quality of marriage and similar dyads. J Marriage \& Fam 1976; 38: 15-28.

31. Piotrowski C. Use of tests and measures in marital and family research. Psychological Reports 1999; 84: 1251-2.

32. Spanier GB. Dyadic Adjustment Scale Manual. North Tonawanda, NY: Multi-Health Systems. 1989.

33. Smilkstein G. The family APGAR: a proposal for a family function test and its use by physicians. J Fam Pract 1978; 6: 1231-9.

34. SPSS 11.0.1. Spss Inc 1989-2001.

35. STATA 8.0, College St, Texas.

36. Toledo V, de la Barra F, López C, George M, Rodríguez J. Pesquisa de problemas en salud 
mental en escolares de $1^{\text {er }}$ ciclo básico en el área occidente de santiago. Rev Chil Neuropsiquiatría 1997; 35: 17-24.

37. Beardslee Wr, Keller MB, LaVori PW, Klerman GK, Dorer DJ, Samuelson H. Psychiatric disorder in adolescent offpring of parent with affective disorders in a non-refered sample. J Affect Disord 15: 313:-322.

38. Grigoroiu-Sebanescu M, Christodorescu D, MagureANU S. Adolescent offspring of endogenous unipolar depressive parents and normal parents. J Affect Disord 1991; 21: 185-98.

39. Weissman MM, Fendrich M, Warner V, WickramaratNE P. Incidence of psychiatric disorder in offpring at high and low risk for depression. J Am Acad Child and adolesc Psychiatry 1992; 31: 640-8.

40. Beardslee WR, Versage E, Gladstone TR. Children of Affectively ill parents: A review of the past 10 year. J Am Acad Child and adolesc Psychiatry 1998; 37: 1134-41.

41. García R, Montt Me, Cabrera J, Valenzuela C, Sepúlveda J, Almonte C. Trastornos psiquiátricos en hijos de padres bipolares. Rev Chil Neuropsiquiatría 1999; 223-32.

42. Minuchen S. Families and Family Therapy. Cambridge. Harvard Press. 1974.

43. Palazzolli M, Boscolo L, Lecchin G, Parados and counter parados. Nueva York Jasón Aronson. 1978.

44. Dawson G, ashman SB, Panagiotides H, Hessl D, Self J, Yamada E et al. Preschool outcomes of children of depressed mothers: role of maternal behavior, contextual risk, and children's brain activity. Child Dev 2003; 74: 1158-75.

45. Miklowitz DJ, Wisniewski SR, Miyahara S, Otto MW, SACHS GS. Perceived criticism from family members as a predictor of the one-year course of bipolar disorder. Psychiatry Res 2005; 136(2-3): 101-11.

46. Elgar F, Mcgrath P, Waschbusch, Stewart S, Curtis L. Mutual influences on maternal depression and child adjustment problems. Clinical Psychology Review 2004; 24: 441-59.
47. BowlBy J. Attachment theory and its therapeutic implications. Adolesc Psychiatry 1978; 6: 5-33.

48. Sexson SB, Glanville DN, Kaslow NJ. Attachment and depression. Implications for family therapy. Child Adolesc Psychiatr Clin N Am 2001; 10: 465-86.

49. Greenough W, B. J. (1992). Induction of brain structure by experience: substrate for cognitive development. In N. C. Gunnar MR (Ed.), Minnesota Symposia on Child Psychology, Developmental Behavioral Neuroscience. (Vol. 24, pp. 155-200). Hilsdale: Lawrence Erlbaum.

50. Glasser D. Child abuse and neglect and the brain: a review. J Child Psychol Psychiatry 2000; 41: 97-116.

51. RUTTER M. Stress, coping and development: some issues and some questions. J Child Psychol Psychiatry 1981; 22: 323-56.

52. Keller MB, Beardslee WR, Dorer DJ. Impact of severity and chronicity of parental affective illness on adaptative functioning and psychopatology in children. Arch Gen Psychiatry 1986; 43: 930-7.

53. Hammen C, Brennan PA. Sevetity, chronicity and timing of maternal depression and risk for adolescent offpring diagnoses in a community sample. Arch Gen Psychiatry 2003; 60: 253-8.

54. Frye AA, Garber J. The relations among maternal depression, maternal criticism, and adolescents' externalizing and internalizing symptoms. J Abnorm Child Psychol 2005; 33: 1-11.

55. Kendler K, Prescott C. A population based twin study of lifetime depression in men and women. Archives of General Psychiatry 1999; 50: 789-96.

56. Lavoine F, Hodgings S. Mental disorders among children with one parent with a life time diagnosis of major depression. En: A Critical Review of the Literature on Children at risk for Major Affective Disorders. Hodgins S, Lapalme C y col. Eds. Ottawa. The Strategic Fund for Childrens Mental Health, 1994, pp 37-82.

57. Beardslee R, Gladstone T, Wright E, Cooper A. A family-Based Approach to Prevention of Depressive Symtoms in Children at Risk: Evidence of parental and Child Change. Pediatrics 2003; 112: 119-31. 